\title{
Characterization of ion Coulomb crystals in a linear Paul trap
}

\author{
K. Okada, ${ }^{1,{ }^{*}}$ M. Wada,${ }^{2}$ T. Takayanagi, ${ }^{1}$ S. Ohtani,${ }^{3}$ and H. A. Schuessler ${ }^{4}$ \\ ${ }^{1}$ Department of Physics, Sophia University, 7-1 Kioicho, Chiyoda, Tokyo 102-8554, Japan \\ ${ }^{2}$ Atomic Physics Laboratory, RIKEN, 2-1 Hirosawa, Wako, Saitama 351-0198, Japan \\ ${ }^{3}$ Institute for Laser Science (ILS), University of Electro-Communications, 1-5-1 Chofugaoka, Chofu, Tokyo, 182-8585, Japan \\ ${ }^{4}$ Department of Physics, Texas A\&M University, College Station, Texas 77843, USA
}

(Received 30 September 2009; published 28 January 2010)

\begin{abstract}
We describe a simple and fast method for simulating observed images of ion Coulomb crystals. In doing so, cold elastic collisions between Coulomb crystals and virtual very light atoms are implemented in a molecular dynamics (MD) simulation code. Such an approach reproduces the observed images of Coulomb crystals by obtaining density plots of the statistics of existence of each ion. The simple method has the advantage of short computing time in comparison with previous calculation methods. As a demonstration of the simulation, the formation of a planar Coulomb crystal with a small number of ions has been investigated in detail in a linear ion trap both experimentally and by simulation. However, also large Coulomb crystals including up to 1400 ions have been photographed and simulated to extract the secular temperature and the number of ions. For mediumsized crystals, a comparison between experiments and calculations has been performed. Moreover, an MD simulation of the sympathetic cooling of small molecular ions was performed in order to test the possibility of extracting the temperature and the number of refrigerated molecular ions from crystal images of laser-cooled ions. Such information is basic to studying ultracold ion-molecule reactions using ion Coulomb crystals including sympathetically cooled molecular ions.
\end{abstract}

DOI: $10.1103 /$ PhysRevA.81.013420

PACS number(s): 37.10.Ty, 37.10.Rs, 52.27.Jt, 42.50.Dv

\section{INTRODUCTION}

Coulomb crystals of laser-cooled ions are being increasingly applied to growing new research fields, i.e., quantuminformation processing and quantum calculations [1-3], as well as to conventional spectroscopic applications [4-7]. Moreover, the sympathetic cooling method has expanded to research applications in cold ion-molecule chemistry and to high-precision molecular spectroscopy. Translationally cold molecular samples are easily produced by sympathetic cooling and photochemical reactions. Photodissociation processes can be studied in detail with single-ion spectroscopy [8,9]. Recently, rovibrational spectroscopy of sympathetically cooled $\mathrm{HD}^{+}$ions in Coulomb crystals has been demonstrated at very low temperatures, when combining Coulomb crystals with a resonance-enhanced multiphoton dissociation technique [10]. Such precision spectroscopy paves the way to testing a possible time dependence of the proton-electron mass ratio [11].

In a different approach, cold neutral molecules have been produced by various other techniques, such as buffer gas cooling [12], photoassociation and magnetic Feshbach resonances of cold alkali atoms [13,14], a Stark decelerator, and a Stark velocity selector $[15,16]$. Electrostatic trapping of polar molecules has also been realized; however, the lifetime is still short $[17,18]$. These prepared cold molecules can be applied to high-precision spectroscopy of neutral molecules, to the study of chemical processes in interstellar media, and toward the demonstration of Bose-Einstein condensation of molecules. Recently a cold collision process between cold polar $\mathrm{CH}_{3} \mathrm{~F}$ molecules, which were produced by a Stark velocity selector, and $\mathrm{Ca}^{+}$Coulomb crystals was investigated with single-ion sensitivity, and ion-molecule reaction rates at low temperatures

*okada-k@sophia.ac.jp were estimated [19]. This new experimental approach can be extended to the study of cold collisions of sympathetically cooled molecular ions and cold polar molecules, which are abundant in interstellar chemistry, and for which many of the reaction rates are unknown [20]. As a prerequisite for the investigation of the reaction rate coefficients of such ultracold ion-molecule collisions by the above methods, one has to know the properties of ion Coulomb crystals including translational temperatures and the numbers of ions (or the number density).

During the past few decades, many simulation studies for ion Coulomb crystals in ion traps were performed by the molecular dynamics (MD) approach in order to study the properties and the structures of the Coulomb crystals [21-27]. For an ionic system in a linear Paul trap, the phase transition as a function of the anisotropy between axial and radial trapping potentials was studied in detail [21]. Then a theoretical investigation of the structural phase transition in a trapped Coulomb crystal was carried out, and the phase diagram as parameters of the anisotropy and the number of ions was given [28]. A scaling law of the criterion for producing planar Coulomb crystal is also given as

$$
\alpha_{3}\left(N_{\text {ion }}\right)=\left(96 N_{\text {ion }} / \pi^{3} w_{1}^{3}\right)^{1 / 2},
$$

where $w_{1}=1.11 \ldots$ is the first two-dimensional transition value from the three-dimensional Coulomb crystals [28]. Recently, MD simulations in a linear multipole trap as well as a linear Paul trap were extensively performed in order to search for the experimental conditions to produce planar or ring-type Coulomb crystals, which might be applicable to quantum-information processing, quantum simulations, and quantum calculations [25-27]. Apart from the studies of such special forms of Coulomb crystals, some simulations were carried out to extract the properties, such as ion temperatures and the number of ions, from experimentally produced 
Coulomb crystals in a linear Paul trap [23,24]. For example, Zhang et al. succeeded in determining the number and the secular temperatures of prolate ion crystals by comparing experimentally observed CCD images to simulated ones [24]. Although the simulation method works well in reproducing Coulomb crystal images, it appears to be complex to choose appropriate values of heating (velocity kick) and cooling parameters (damping force) in the equations of motion.

In this paper, we report a simple and fast method of simulating observed images of ion Coulomb crystals in a linear Paul trap. By introducing cold elastic collisions between stored ions and virtual very light atoms in our original MD simulation code [25], CCD images of Coulomb crystals are successfully reproduced by the density plot of the statistics of existence of each ion. Then the important properties of Coulomb crystals such as ion temperatures, structures of ion Coulomb crystals, and the number of ions were extracted from the CCD images with the help of the MD simulation.

In short, we numerically solve Newton's equations of motion for all trapped ions under the pseudopotential approximation using the velocity Verlet algorithm [29]. After each integration step, the standard equation is applied to the determination of an ion's velocity after a classical elastic collision with a virtual light atom. The method functions well in the reproduction of observed images of Coulomb crystals even though such a collision process is virtual. The reason is as follows. Generally, experimentally produced Coulomb crystals are heated by occasional collisions with the residual background gas at a certain interval. Although the collisional heating effect is very large, the collision interval is long enough compared to the thermalization time of the heated crystal under our experimental conditions (typical exposure time of CCD is $10 \mathrm{~s}$ ) [24]. Since the disturbed ion trajectories by such occasional collisions are time-averaged and the collisional effect on the CCD image is also averaged, it is considered that the observed Coulomb crystals are in the thermal equilibrium, as long as the observation period by a CCD camera is long enough. The concept of our simulation approach is different from the previous methods, because the purpose of the simulation is only to reproduce images of observed Coulomb crystals, which are characterized by a certain equilibrium temperature. Apart from the number of ions, the fitting parameter used to simulate the observed images is only the temperature of the virtual light atoms. This simple simulation method has the advantage of simple programming and short computing time compared to previous calculation methods, in which the time-dependent radiofrequency electric fields, radiation pressure forces depending on an ion velocity and the photon recoil effect [25], or the intentional heating effect ("velocity kick") and a viscous damping force proportional to the ion velocity [24] were considered.

As a demonstration of our method, we simulated the observed Coulomb crystals during the production processes of planar Coulomb crystals in a linear Paul trap, since there is much interest in these applications in the community of the fields of scalable quantum-information processing and quantum computation using ion Coulomb crystals in ion traps $[2,26,27,30]$. In this paper, the experimental result of the systematic transformation of a Coulomb crystal's shape with a definite number of ions is presented, and the images are compared to our simulation results. Observed Coulomb crystals with a large number of ions $(>1000)$ were also compared to the simulation. Finally, the sympathetic crystallization of simple molecular ions is obtained step by step to demonstrate the associated change of the laser-cooled ion images by the secular temperature of laser-cooled ions and the number of molecular ions imbedded in the Coulomb crystal. The details of this effect will be useful in determining the properties of sympathetically crystallized molecular ions.

\section{EXPERIMENTAL SETUP}

The experimental setup is as follows. The linear Paul trap consists of four rod electrodes with a diameter of 8 $\mathrm{mm}$. The distance from the trap center axis to the surface of the electrodes is $r_{0}=3.5 \mathrm{~mm}$. To axially trap ions, the rod electrodes are divided into three sections, and a different static voltage is applied to each section [see Fig. 1(a)]. The length of the center section is $10 \mathrm{~mm}$. The rf voltages are applied to one pair of opposing rods, and the other pair is rf grounded. The typical driving frequency and amplitude of
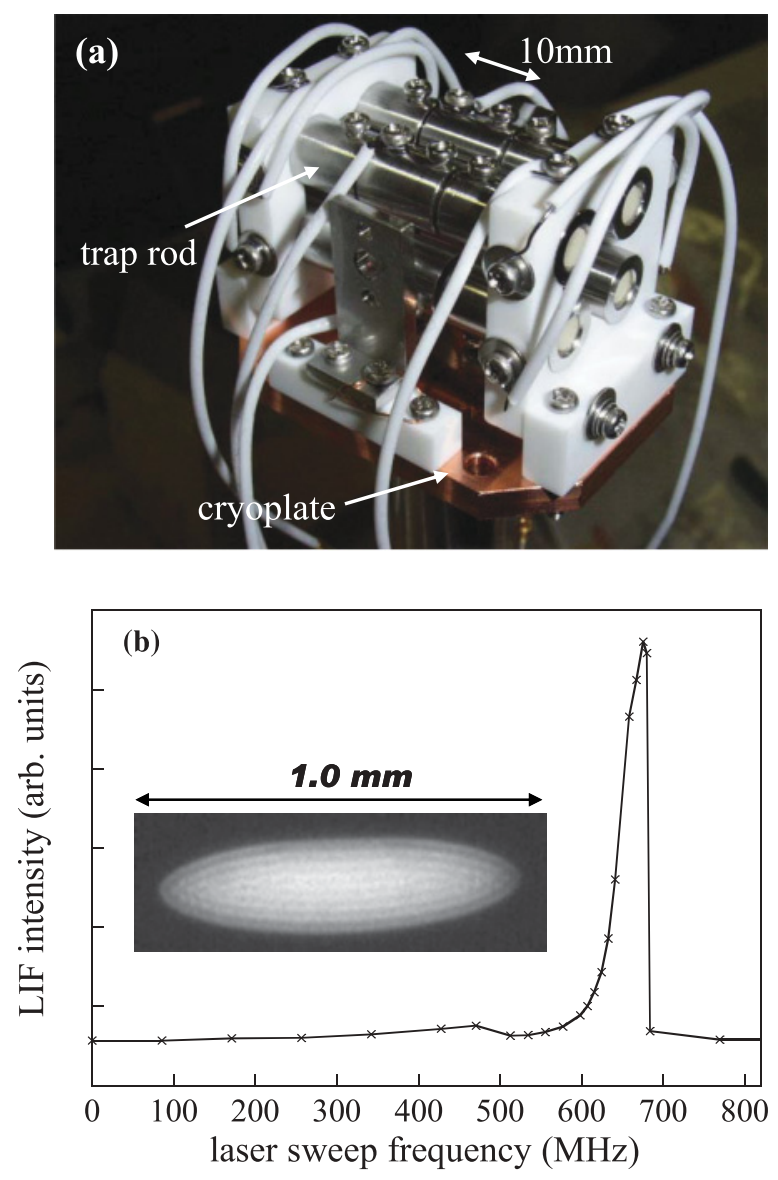

FIG. 1. (Color online) (a) A photograph of the cryogenic linear Paul trap. The rod diameter and the inner radius $\left(r_{0}\right)$ are 8 and $3.5 \mathrm{~mm}$, respectively. The central trap region is $10 \mathrm{~mm}$. (b) A LIF spectrum and a crystal image of the same $\mathrm{Ca}^{+}$crystal. The linewidth of the spectrum is about $30 \mathrm{MHz}$ (half width at half maximum). The static voltage of $V_{z}=3.5 \mathrm{~V}$ was applied to both side sections of the ion trap. The CCD image is observed at about $-10 \mathrm{MHz}$ from the peak of the spectrum. The CCD exposure time is $10 \mathrm{~s}$. 
the linear Paul trap are $\Omega / 2 \pi=5.65 \mathrm{MHz}$ and $V_{\mathrm{ac}}=260 \mathrm{~V}$, respectively. The static voltages of both side sections were changed from $0 \mathrm{~V}$ to $32 \mathrm{~V}$ to manipulate the shapes of the Coulomb crystals as described later. A compact miniature UHV chamber (3.2 in. diameter and 1.38 in. height) enclosing the ion trap is evacuated by an ion pump and a turbo molecular pump backed by a rotary pump. The ion trap is mounted on a cryogenic vessel containing liquid-nitrogen to obtain an ultrahigh vacuum $\left(<10^{-8} \mathrm{~Pa}\right)$.

Two grating stabilized diode lasers $(\lambda=397$ and $866 \mathrm{~nm})$ locked to temperature-controlled optical cavities are used for laser cooling of the $\mathrm{Ca}^{+}$ions [31]. An ion Coulomb crystal consisting of $\mathrm{Ca}^{+}$ions, which emit laser-induced fluorescence (LIF) at $397 \mathrm{~nm}$, is observed by a cooled CCD camera at right angles to the trap axis. The camera with the lens system and a UV filter is mounted on a precision stage outside the vacuum chamber to adjust to the imaging position of the Coulomb crystal. The magnification of the lens system was selected to $6 \times$ or $10 \times$ according to circumstances. The CCD exposure time is typically set to $10 \mathrm{~s}$.

In Fig. 1(b), the result of observations of the LIF spectrum and the image of the same crystal are shown. The CCD image is recorded at about $-10 \mathrm{MHz}$ from the peak of the spectrum. A fluorescence dip in the spectrum indicates the phase transition from the cloud state to the liquid and crystal states, and actually a shell structure is observed as the CCD image. Since the volume of the ion crystal is known from the observed image and by assuming cylindrical symmetry of the crystal, the number of ions in the crystal is estimated to be about 940 using the equation of the number density at $0 \mathrm{~K}$ under the pseudopotential approximation, i.e.,

$$
n_{q}=\frac{\epsilon_{0} V_{\mathrm{ac}}^{2}}{m \Omega^{2} r_{0}^{4}},
$$

where $m$ and $\epsilon_{0}$ are the mass of the ions and the permittivity of the vacuum, respectively. The above equation demonstrates that the number of zero-temperature ions is proportional to the volume of the Coulomb crystal in the linear Paul trap. For relatively large Coulomb crystals like in Fig. 1(b), we roughly estimated the number of ions by this method before starting the MD simulation. A final more precise determination of the number of ions is then possible by direct comparison with the simulation images.

\section{MOLECULAR DYNAMICS SIMULATION}

We carried out MD simulations of the trapped ions to extract the information from the observed Coulomb crystals. Ion trajectories and velocities of all the trapped ions are calculated by solving Newton's equations of motion similar to the method described in Ref. [25]. The difference between the present and the previous method is as follows.

In the present simulation, only time-independent trapping forces by the radial pseudopotential and the axial harmonic potential as well as Coulomb forces between ions are considered. Instead of a radiation pressure force and a heating term such as "velocity kick," cold elastic collisions between trapped ions and virtual very light atoms are introduced. After each integration step of the algorithm, we apply the standard equation for the determination of the ion velocity $\boldsymbol{v}_{\text {ion }}^{\prime}$ after a classical elastic collision [32], i.e.,

$$
\begin{gathered}
\boldsymbol{v}_{\mathrm{ion}}^{\prime}=\frac{m_{\mathrm{gas}}}{m_{\mathrm{ion}}+m_{\mathrm{gas}}} v \boldsymbol{n}_{0}+\frac{m_{\mathrm{ion}} \boldsymbol{v}_{\mathrm{ion}}+m_{\mathrm{gas}} \boldsymbol{v}_{\mathrm{gas}}}{m_{\mathrm{ion}}+m_{\mathrm{gas}}}, \\
v=\left|\boldsymbol{v}_{\mathrm{ion}}-\boldsymbol{v}_{\mathrm{gas}}\right|,
\end{gathered}
$$

where the unit vector $n_{0}$ of the ion velocity after the collision is given by random numbers, and the virtual atom velocity $\boldsymbol{v}_{\text {gas }}$ is randomly generated by the Box-Muller transform at the assumed equilibrium temperature of the ensemble of virtual very light atoms. The mass number of a virtual atom is set to $M_{\text {ion }} / 100$, which is too light to disturb the ion motions in only one collision, and a large number of cold collisions (typically $10^{6}$ ) are introduced to simulate the observed CCD images of the Coulomb crystals. Since all forces are not dependent on ion velocities, the Runge-Kutta algorithm can be replaced by the velocity Verlet algorithm, which is much faster for numerical integrations. The time step of the calculations is selected at a fixed value between 10 and 40 ns. The crystal state is reached when the trajectories of all ions are almost frozen. After that, the location of each ion is recorded as follows. Initially we divide an image area of typically $1.7 \times 1.7 \mathrm{~mm}^{2}$ into many small cells (typically $512 \times 512$ in number, $2.62144 \times$ $10^{5}$ cells), which correspond to the number of pixels of the CCD camera, and the content of each cell is set to 0 . Then, at each integration step, we increment a cell counter by one if an ion exists in this cell. The number of integration steps is typically set to $10^{6}$. In this way the CCD images of the Coulomb crystals are reproduced by the density plot of the cells.

The reasons that the present calculation method works well are as follows. Experimentally produced Coulomb crystals are normally heated by occasional collisions with the residual background gas at a certain interval as well as by the rf heating effect, which is negligible for very cold ion samples [33]. The mean free time between collisions in $10^{-8} \mathrm{~Pa}$ of $\mathrm{H}_{2}$ gas is estimated to $\sim 3$ s from the Langevin rate [34], when a Coulomb crystal consists of $100 \mathrm{Ca}^{+}$ions. Although the collisional heating effect is very large, the collision interval is long enough compared to the thermalization time of the heated crystal under our experimental conditions [24]. Since the ion trajectories are time-averaged during the observation period (CCD exposure time), it can be considered that the Coulomb crystals are in thermal equilibrium as mentioned in Sec. I. In the present calculation method, this time average is replaced by a large number of virtual cold collisions with virtual very light atoms (invoking the ergodic hypothesis). In this way, we take into account the rare occasional collisional heating effect as well as the cooling effect at the same time. Moreover, the following reasons support the assumptions. (1) Since the kinetic energy of an experimentally produced Coulomb crystal falls into near the bottom of the pseudopotential well, the pseudopotential approximation holds for Coulomb crystals under the present experimental conditions. (2) The small micromotion effect on a structure of a Coulomb crystal is averaged out on the CCD images, which are integrated for several seconds. Even if for large ion crystals, drift motions exist, they are also averaged out. Actually, the presented photographs represent time-averaged positions of ions. 


\section{RESULTS AND DISCUSSIONS}

\section{A. Determination of the geometrical factor}

For the axial confinement of ions in the linear Paul trap, a static voltage $V_{z}$ is applied to the outside sets of segmented electrodes. In the present simulations, the static potential near the middle of the trap is assumed to be

$$
\phi_{\mathrm{s}}(x, y, z)=\frac{\kappa V_{z}}{z_{0}^{2}}\left(z^{2}-\frac{x^{2}+y^{2}}{2}\right),
$$

where $2 z_{0}$ is the length of the center part of the segmented trap, and $\kappa$ is the geometrical factor which is determined by the particular trap geometry. Initially the geometrical factor near the center along the trap axis ( $z$ axis) was evaluated to $\kappa \sim 0.32$ from the numerical values of the electric potential calculated by the SIMION 3D code [35]. However, we know empirically that the experimental $\kappa$ is different from the numerically evaluated one particularly for low $V_{z}$. Since to our knowledge this effect has not been described in past simulations, it is worth demonstrating it. As expected from Eq. (2), the shape of the crystal, i.e., the aspect ratio, depends on the number of ions and on the depth of the trapping potentials at very low temperatures. Thus we have experimentally determined $\kappa$ by comparing observed CCD images with simulation images of a Coulomb crystal.

The demonstration for Coulomb crystals consisting of $30 \mathrm{Ca}^{+}$ions is shown in Fig. 2. The MD simulations were performed under the same condition as the experiment by systematically changing $\kappa$ [Figs. 2(a')-2(d')]. Then, the vertical and horizontal lengths of the simulated images were compared to those of the corresponding CCD images. The $\kappa$ value obtained in this way as a function of $V_{z}$ is depicted in the lower graph of Fig. 2. In this measurement, $\kappa$ decreases to a lower value than the numerical one at small $V_{z}$. The reasons are attributed to contact potentials as well as stray static electric fields, which arise from slightly charged insulators at both sides of the ion trap. It is shown that $\kappa$ is close to the numerical value for $V_{z}$ larger than $15 \mathrm{~V}$, since the weak stray fields are masked by the strong fields for large $V_{z}$. In the following MD simulations, we used the appropriate values of $\kappa\left(V_{z}\right)$ obtained by this approach.

\section{B. Formation process of planar Coulomb crystals}

To our knowledge, there is only one experimental demonstration of planar Coulomb crystals in linear Paul traps [36], although such a system has been given much attention in the field of quantum calculations [2,26,27]. In the previous experiment [36], small planar crystals of up to 19 ions have been produced and were observed as images by a CCD camera. Since the planar crystals were observed at an angle of about $45^{\circ}$ with respect to the trap axis, some of the ions were out of the focal plane of the image system, and it appeared to be difficult to extract the actual size of the crystals and compare them with the simulation images. In addition, the production process of the planar crystal and the systematic changes of the images by the anisotropic parameter of the trapping potential were not observed. In our experiment, we chose a right angle with respect to the trap axis for observation to avoid the distortion of the images. Such distortions would be large if we were to tilt
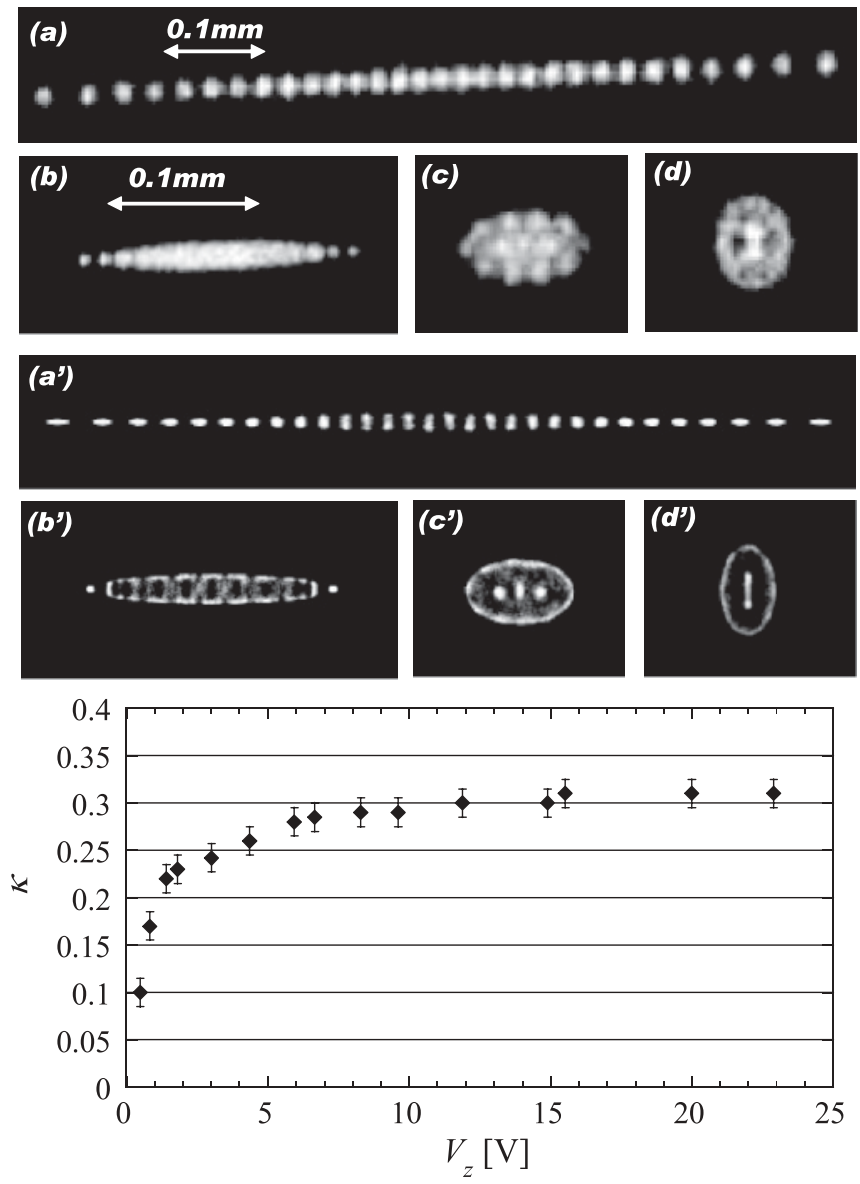

FIG. 2. Experimental determination of the geometrical factor $\kappa$ in Eq. (4). The observed images (a)-(d) show the ion Coulomb crystals including $30 \mathrm{Ca}^{+}$ions at $V_{z}=$ (a) 0.5 , (b) 1.8 , (c) 8.3 , and (d) $14.9 \mathrm{~V}$, respectively. The corresponding simulation images are shown in (a')-(d'), where $\kappa=$ (a') 0.10, (b') 0.23, (c') 0.29, and (d') 0.30 , respectively. All scales of the images are the same in the figure. In the simulation, the temperature of the virtual light atoms was set to $10 \mathrm{mK}$. The rf frequency and the amplitude are $f_{\text {rf }}=5.634 \mathrm{MHz}$ and $V_{\mathrm{ac}}=252.5 \mathrm{~V}$, respectively. The lower graph shows a plot of $\kappa$ evaluated from the CCD images versus $V_{z}$. The error bar is estimated to be 0.015 of the difference between the experimental and the simulated images for large $V_{z}$.

the observation angle due to the limiting size and the thickness of the available view port.

The formation process of a planar Coulomb crystal with $N_{\text {ion }}=34$ was investigated in detail (Fig. 3). In this experiment, we have sequentially observed the same crystal by changing the axial static voltages from $30 \mathrm{~V}$ to $0.6 \mathrm{~V}$ to avoid losses of ions due to an increase of $V_{z}$. Since the crystal structures can be clearly distinguished, the temperatures of the observed ion crystals can be determined by comparison with the simulated images. The selected results at $V_{z}=1.0,3.8$, and 20.0 V are shown in Fig. 4. The top and the lower images depict the experimental and simulated images, respectively. In the simulated images, the varying temperatures of the virtual very light buffer gas are indicated. The temperatures of the ion crystals at $V_{z}=1.0,3.8$, and $20.0 \mathrm{~V}$ are determined to be $T_{\text {ion }}=3 \pm 1,5 \pm 1$, and $8 \pm 2 \mathrm{mK}$, respectively. As expected, a larger $V_{z}$ gives a larger average temperature of the ion crystal. 


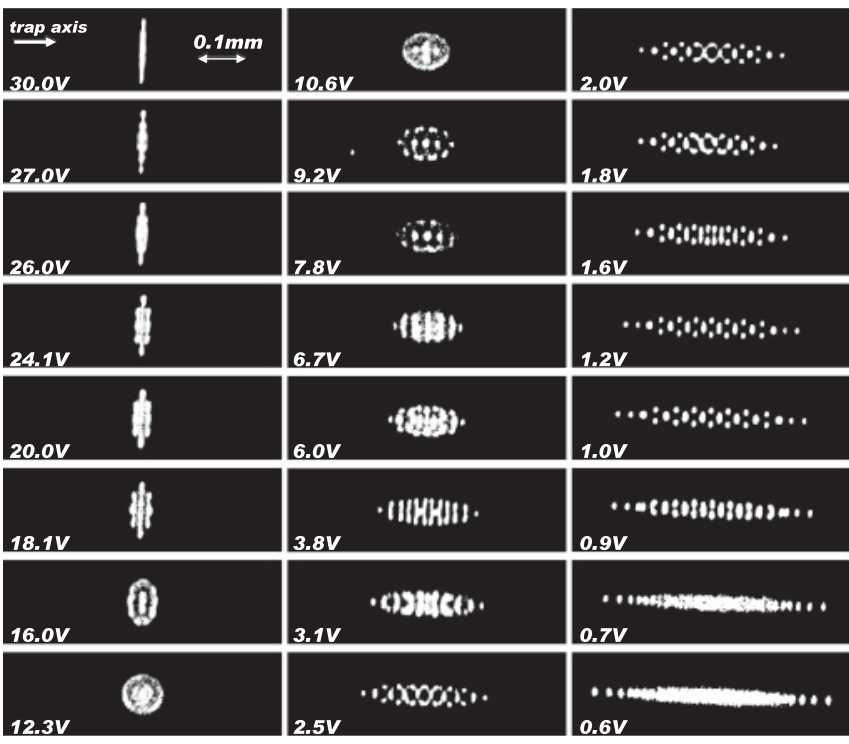

FIG. 3. Systematic observation of ion Coulomb crystals of $34 \mathrm{Ca}^{+}$ions as a function of the axial static voltage $V_{z}$. The listed voltage in each image indicates the applied $V_{z}$. The rf frequency and the amplitude are $f_{\mathrm{rf}}=5.634 \mathrm{MHz}$ and $V_{\mathrm{ac}}=255.5 \mathrm{~V}$, respectively.

This is due to the increase of the micromotion amplitudes of the outer ions, because it promotes the collisional heating effect with the background gas. As shown in Fig. 5, we have also performed similar observations for other numbers of ions.

In order to determine the two-dimensional structure of the stick-like image of Fig. 3 at $V_{z}=30 \mathrm{~V}$, we have performed the MD simulation under the same condition as in the experiment. Since the horizontal direction is parallel to the trap axis, the image suggests that a planar Coulomb crystal is produced.

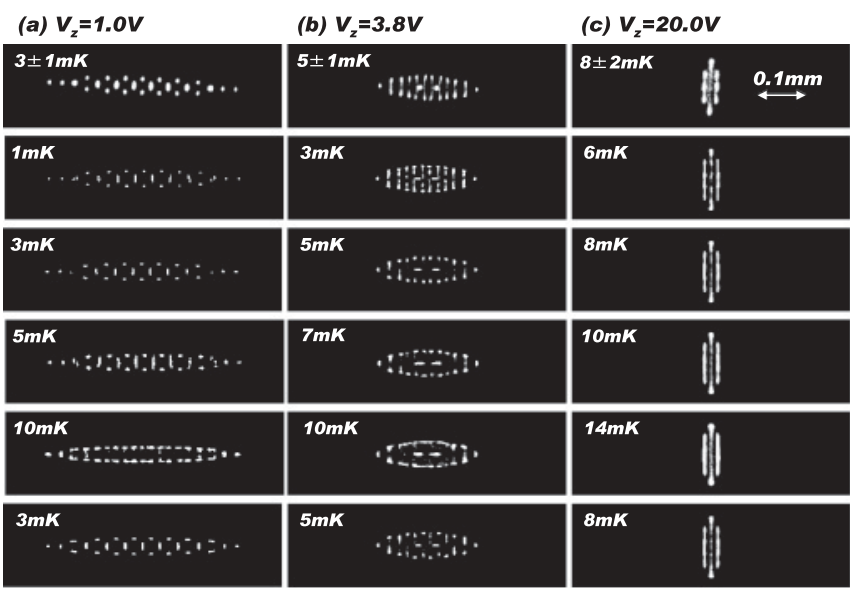

FIG. 4. Determination of the temperatures of the observed ion Coulomb crystal with 34 ions by the MD simulation. For each selected $V_{z}$, the uppermost and the lower images show the CCD image and the simulated ones, respectively. The temperatures are determined by comparing the observed images with the simulation images for a definite temperature of the virtual atomic buffer gas. The best fit temperatures are indicated on the top experimental images. There are two possible observation angles different by $90^{\circ}$ in a linear Paul trap. If one observes the crystals of (a)-(c) from the other direction, the bottom images should be observed.

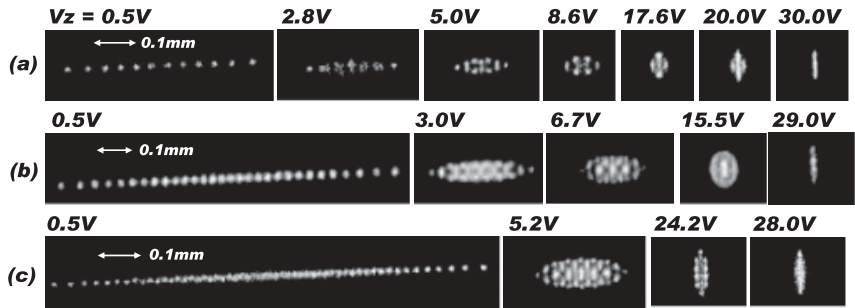

FIG. 5. Systematic change of the shape of observed Coulomb crystals of (a) $N_{\text {ion }}=12$, (b) 30, and (c) 55 as a function of the axial static voltage $V_{z}$. Planar Coulomb crystals were successfully produced in (a) and (b). In the case of (c), the number of ions in the crystal reduced to 34 ions as $V_{z}$ increased up to $28 \mathrm{~V}$ (see also Fig. 9).

Before starting the simulation, the temperature of the crystal should be determined. However, it is difficult to precisely determine the temperature of the stick-like image in Fig. 3, since a change of the image caused by an ion temperature variation is small. Thus we have initially safely set the upper limit to $T_{\text {ion }} \sim 14 \mathrm{mK}$ based on the comparison with simulated images and the trend of the increase of $T_{\text {ion }}$ by $V_{z}$ displayed in Fig. 4.

Figure 6 shows the simulation results at selected temperatures. When the ion temperature is higher than $3 \mathrm{mK}$, the ions form a multiple-ring structure. It is likely that the ions inside the rings move freely and cannot jump over to the other rings. In this case, the structure of the crystal is similar to the one observed in Penning traps [37]. If the ions are cooled to less than $1 \mathrm{mK}$, the ions are localized around a well-defined position. A notable feature is that the central position of the crystal becomes vacant at a temperature lower than $\sim 1 \mathrm{mK}$, while it tends to be occupied at the higher temperatures. The simulation results suggest that the ions must be cooled to a lower temperature than $\sim 1 \mathrm{mK}$ in order to be nonrotating and localized in a planar Coulomb crystal.

Figure 7 shows CCD images of relatively large ion crystals including initially (a) 290 and (b) 1400 ions, respectively. The indicated voltage on each picture corresponds to the axial static voltage $V_{z}$. To determine the number of ions $\left(N_{\text {ion }}\right)$ included

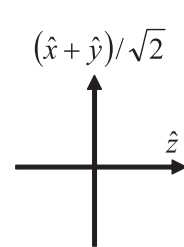

$14 m K$

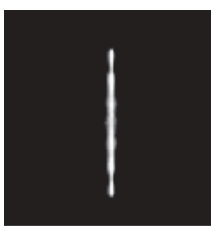

${ } \boldsymbol{\hat { x }}^{\hat{y}}$

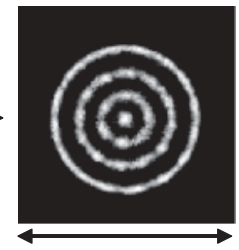

$0.24 \mathrm{~mm}$
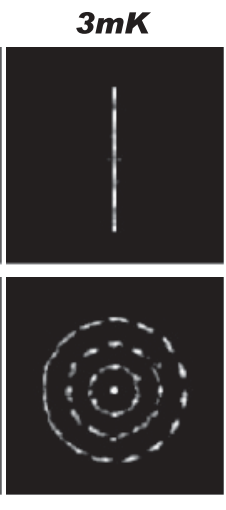
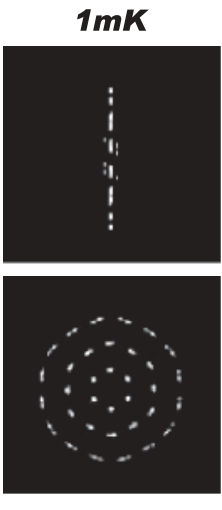

FIG. 6. Simulated images under the same condition as the experiment in Fig. 3 at $V_{z}=30 \mathrm{~V}$. The temperatures of the virtual light atoms are indicated above the picture. The coordinates shown on the left correspond to the images to the right of them. 
(a) $N_{\text {ion }}=\mathbf{2 9 0}$
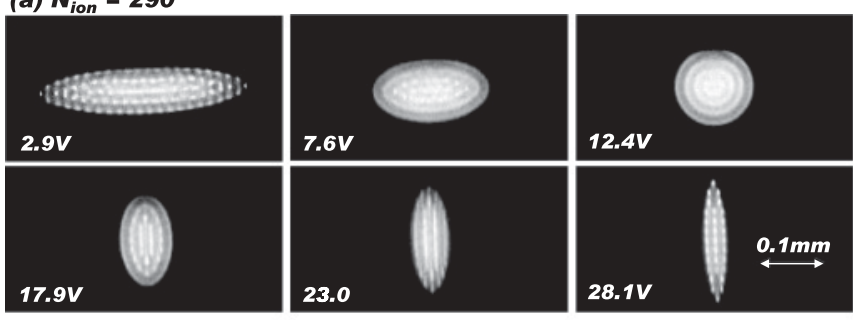

(b) $N_{\text {ion }}=1400$
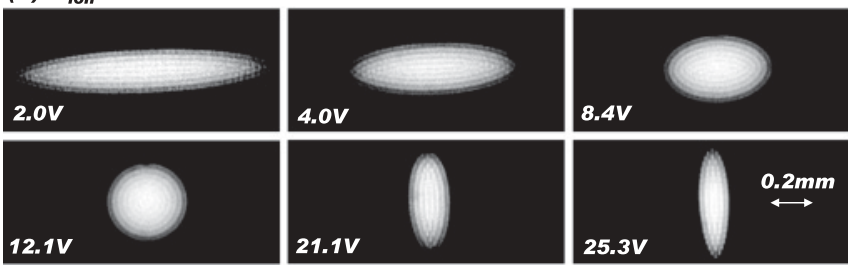

FIG. 7. Observed ion crystals of (a) $N_{\text {ion }}=290$ and (b) $N_{\text {ion }}=$ 1400 at low $V_{z}$. The indicated voltage in each frame is the applied $V_{z}$. The conditions of the applied rf voltages are (a) $f_{\mathrm{rf}}=5.634 \mathrm{MHz}$, $V_{\mathrm{ac}}=253.5 \mathrm{~V}$, and (b) $f_{\mathrm{rf}}=5.65 \mathrm{MHz}, V_{\mathrm{ac}}=263.5 \mathrm{~V}$, respectively.

in the crystals, the following procedure was carried out. To begin with, we estimate $N_{\text {ion }}$ using Eq. (2) and perform the MD simulation under the same condition as in the experiment. Then we compare the volume of the simulated image to that of the CCD images. Since Eq. (2) suggests $N_{\text {ion }}$ is proportional to the volume of the ion crystal, the number of ions for the MD simulation can be corrected by multiplying the initial $N_{\text {ion }}$ with the ratio to the initial and observed crystal volumes. After repeating the same procedure several times, $N_{\text {ion }}$ can be determined for the present condition. By being based on this $N_{\text {ion }}$ and the ratio of crystal volume, we determine the number of ions of the ion crystals at different $V_{z}$. The results are shown in Figs. 8(a) and 8(b).

Although the number of ions contained in the Coulomb crystal is constant up to $V_{z} \sim 18 \mathrm{~V}$, it gradually reduces when increasing the axial static voltage to larger values. These larger crystals do not quite form a planar shape when applying the
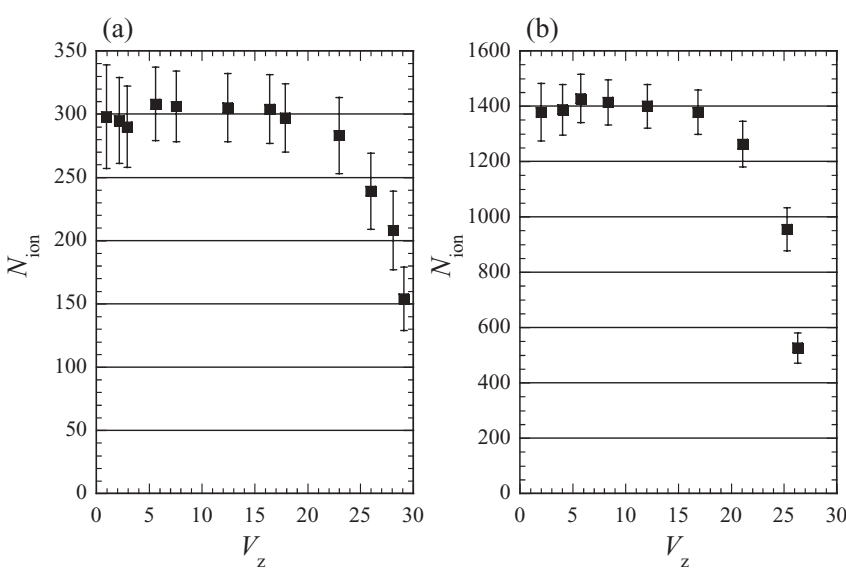

FIG. 8. Plots of the number of ions in the observed Coulomb crystals of Fig. 7 as a function of $V_{z}$. The error bar of each point is caused by the uncertainty of the determination of the edge of the crystal image. The unit of a horizontal axis is Volt.

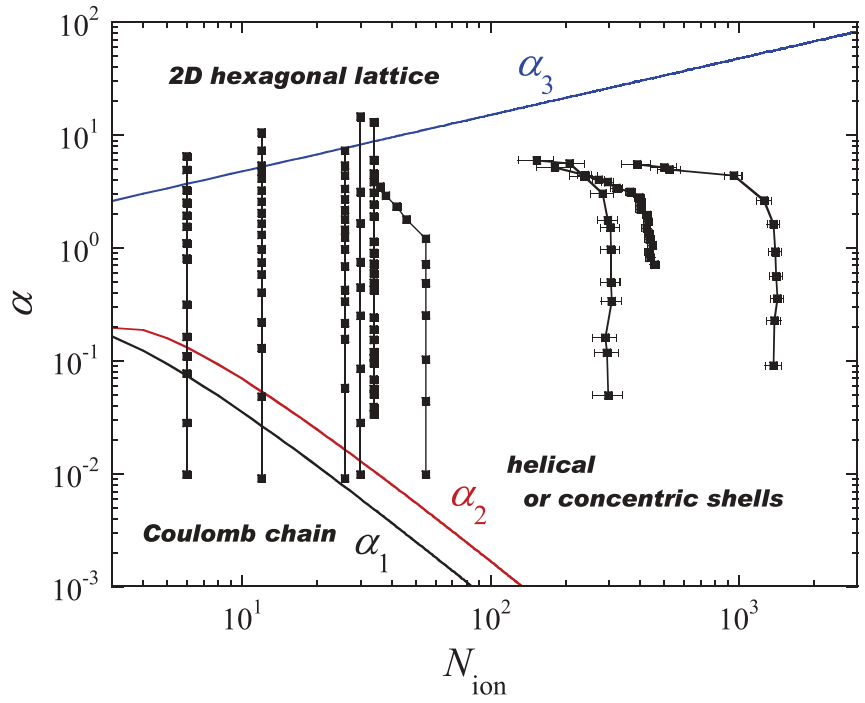

FIG. 9. (Color online) Structural phase diagram of cold charged particles in a harmonic potential [28]. The ratio of the depth of the potential wells between the axial and the radial directions are plotted as a function of the number of ions, where $\alpha=\omega_{z}^{2} / \omega_{r}^{\prime 2}$ and $\omega_{r}^{\prime}=$ $\sqrt{\omega_{r}^{2}-\omega_{z}^{2} / 2}$. Our observation conditions are shown as the points. Connected points indicate the transformation of a crystal to different structures by varying $V_{z}$ and in this way $\alpha$.

similar $V_{z}$ at which a planar crystal for a small number of ions is produced. This is because the sum energy of the Coulomb repulsion between ions is much larger than that of the smaller crystals. When the axial voltages increased to $30 \mathrm{~V}$ or more, the number of ions rapidly decreased to a few dozen ions. Then the planar crystal of such a small number of ions was produced as shown in Figs. 5(a)-5(c).

The present observations of ion Coulomb crystals are summarized by a structural phase diagram of a non-neutral plasma in a harmonic potential [28]. As shown in Fig. 9, the ratio of the depth of the potential wells between the axial and the radial directions, $\alpha=\omega_{z}^{2} / \omega_{r}^{\prime 2}$, are plotted as a function of the number of ions, where we used the corrected radial secular frequency given as [38]

$$
\omega_{r}^{\prime}=\sqrt{\omega_{r}^{2}-\omega_{z}^{2} / 2} .
$$

The lines given by Dubin [28] indicate the boundary for structural phase transitions of a Coulomb crystal: a Coulomb chain $\left(\alpha<\alpha_{1}\right)$, an inhomogeneous zigzag crystal $\left(\alpha_{1}<\alpha<\alpha_{2}\right)$, a helical structure or a crystal with concentric shells $\left(\alpha_{2}<\right.$ $\left.\alpha<\alpha_{3}\right)$, and a planar crystal with a hexagonal lattice $\left(\alpha>\alpha_{3}\right)$. Since this phase diagram is known to match mainly simulation results of large $N_{\text {ion }}$ [28], it is not relevant to fit to the experimental results of small ion crystals such as Figs. 3 and 5. Actually, it is expected that, in this case, the observed planar crystals have multiple-ring structures instead of twodimensional hexagonal lattice structures. Similar results were reported in previous experimental work [36] as well as in a simulation study [27].

In contrast to the above small- and medium-size crystals, for relatively large ion crystals of $N_{\text {ion }}>100$, planar shapes could not be observed. As shown in Fig. 8, the number of ions decreased with increasing $\alpha$. One of the reasons for the 
decrease can be attributed to low laser powers, because the rf heating effect, which promotes the collision heating effect, is larger for the ions placed near the edge of the crystal. Second, there are imperfections of the trap arrangement, which give small distortions of the electric fields inside the ion trap. The outer ions are more sensitive to the patch effect of electric charges. Figure 9 suggests that a lattice structure of such large planar crystals is hard to produce using the present linear Paul trap. This is due to the intrinsic reason, that $\omega_{r}$ decreases with increasing $\omega_{z}$ according to Eq. (5). Thus the radial potential becomes quickly shallow as $V_{z}$ increases, and the above undesirable effects prevent the Coulomb crystals from forming a planar structure. Actually there is a limit at $V_{z} \approx 33.5 \mathrm{~V}$ to maintain a positive pseudopotential under the present condition of applied rf fields and electrode arrangement.

\section{Determination of the ion temperature}

As a demonstration of obtaining temperature information, we have determined the ion temperatures of the selected CCD images of Fig. 7. The simulated images of Fig. 10 correspond to the ones observed at the lowest $V_{z}$ of Fig. 7. Since many single spots in the concentric ellipsoidal shells are seen in the observed crystal consisting of $290 \mathrm{Ca}^{+}$ions, the temperature of the crystal should be less than $7 \mathrm{mK}$ but higher than $5 \mathrm{mK}$; that is, the best fit temperature is $6 \pm 1 \mathrm{mK}$. In the case of the larger ion crystal of $N_{\text {ion }}=1400$, the individual spots are observed only at the edges of the ion crystal, and each shell is well resolved. By comparison to the simulation, the fitted temperature is achieved for $\sim 7 \mathrm{mK}$. It is to be noted that the ion temperature determined by CCD images normally gives an

(a) $N_{\text {ion }}=290, V_{z}=2.9 \mathrm{~V}$
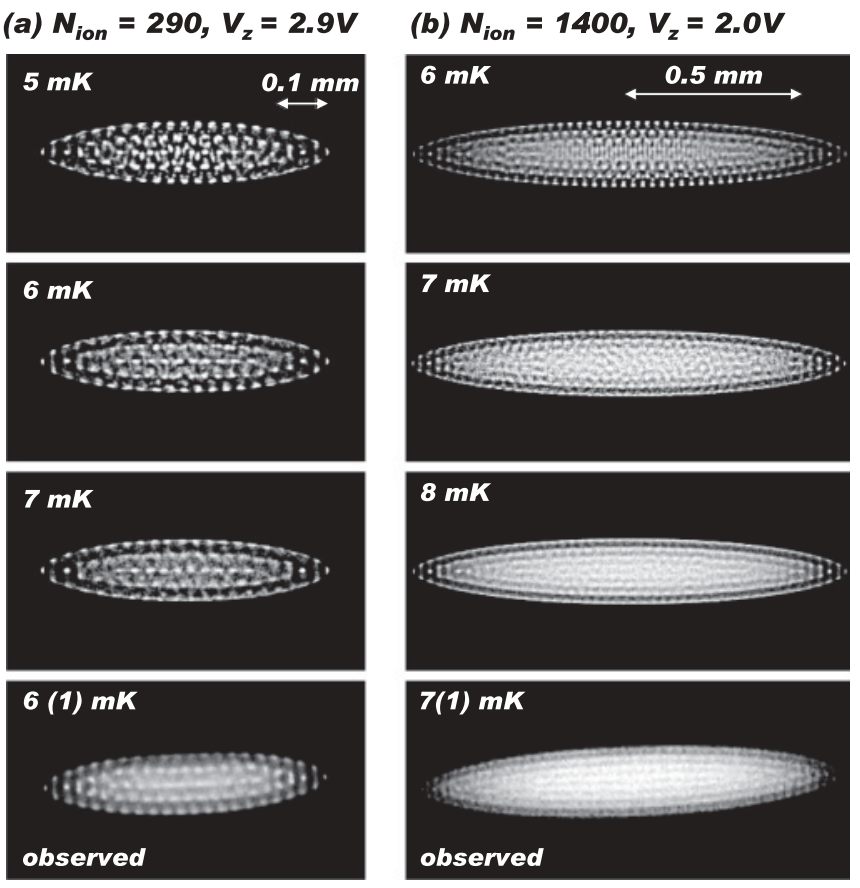

FIG. 10. Determination of temperatures of ion Coulomb crystals with $N_{\text {ion }}=290$ and 1400 observed at the lowest $V_{z}$ in Fig. 7. The simulation conditions are the same as the experiment. The best fit temperatures are $6 \pm 1$ and $7 \pm 1 \mathrm{mK}$ for Figs. 7(a) and 7(b), respectively.

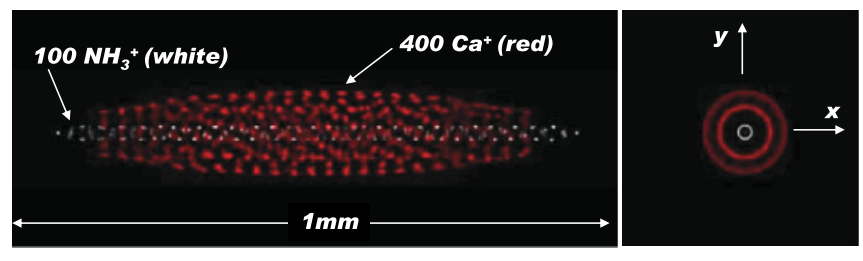

FIG. 11. (Color online) Simulation images of 100 sympathetic crystallization of $\mathrm{NH}_{3}{ }^{+}$ions by 400 laser-cooled ${ }^{40} \mathrm{Ca}^{+}$ions. The temperature of the virtual light atoms for cooling of $\mathrm{Ca}^{+}$is set to $6.0 \mathrm{mK}$. The average energies of $\mathrm{Ca}^{+}$and $\mathrm{NH}_{3}{ }^{+}$ions are $6.0(3)$ and $6.4(1.2) \mathrm{mK}$, respectively, where the values in the parentheses indicate the standard deviations. The $\mathrm{rf}$ and the axial static voltages are $f_{\mathrm{rf}}=5.65 \mathrm{MHz}, V_{\mathrm{ac}}=250 \mathrm{~V}$, and $V_{z}=2.0 \mathrm{~V}$, respectively.

upper bound value, because the imaging optics has a limited spatial resolution. However, it is clear that the resolution of the present CCD images is good enough to distinguish a temperature difference of $\Delta T_{\text {ion }} \sim 1 \mathrm{mK}$.

\section{MD simulation of sympathetic cooling}

It is interesting to apply crystallized molecular ions as a target for studying ultracold ion-molecule collisions at millikelvin temperatures. With this in mind, we have applied the present simulation method to the sympathetic cooling of simple molecular ions, namely, $\mathrm{NH}_{3}{ }^{+}$, as an example. In order to perform sympathetic cooling, in addition to collisions with virtual very light atoms which simulates the conditions of laser-cooled ions, now also the additional cooling of trapped molecular ions must be achieved by the Coulomb interaction with cooled $\mathrm{Ca}^{+}$ions.

In Fig. 11, we show a simulation image of the sympathetic crystallization of 100 light $\mathrm{NH}_{3}{ }^{+}$ions by laser-cooled 400 heavy ${ }^{40} \mathrm{Ca}^{+}$ions. We can successfully simulate the sympathetic crystallization of $\mathrm{NH}_{3}{ }^{+}$ions using the present method. As expected, the light $\mathrm{NH}_{3}{ }^{+}$ions are distributed inside the $\mathrm{Ca}^{+}$ crystal due to the $1 / M_{\text {ion }}$ dependence of the pseudopotential of a linear Paul trap. The average energies of $\mathrm{Ca}^{+}$and $\mathrm{NH}_{3}{ }^{+}$ ions are $6.0(3)$ and $6.4(1.2) \mathrm{mK}$, respectively, where the temperature of virtual light atoms is set to $6.0 \mathrm{mK}$, and the values in the parentheses indicate the standard deviations. It implies the temperatures coincide within the uncertainties. In an actual experiment, only the $\mathrm{Ca}^{+}$crystal can be observed by laser-induced fluorescence. However, the image of the $\mathrm{Ca}^{+}$ crystal is influenced by the presence of $\mathrm{NH}_{3}{ }^{+}$ions. If the change of the $\mathrm{Ca}^{+}$image caused by the effect of the number of $\mathrm{NH}_{3}{ }^{+}$ions present and of the temperature of ions is detected, it is possible to use sympathetically cooled $\mathrm{NH}_{3}{ }^{+}$ions as an ultracold target in a collision experiment. For this purpose, we have performed an MD simulation by systematically changing the number of sympathetically cooled molecular ions $\left(N_{\mathrm{SC}}\right)$, the number of laser-cooled ions $\left(N_{\mathrm{LC}}\right)$, and the temperature of laser-cooled ions $\left(T_{\mathrm{LC}}\right)$.

In Fig. 12, we show simulated images for the sympathetic cooling for different numbers of $\mathrm{NH}_{3}{ }^{+}$ions under the influence of a fixed number of $\mathrm{Ca}^{+}$ions. In Fig. 12(a), the molecular ions are crystallized at least up to $N_{\mathrm{SC}}=80$. The difference of the $\mathrm{Ca}^{+}$crystal by the increase of $N_{\mathrm{SC}}$ is clearly shown. At smaller numbers of $\mathrm{NH}_{3}{ }^{+}$ions $\left(N_{\mathrm{SC}}<40\right)$, the temperature $T_{\mathrm{SC}}$ is 

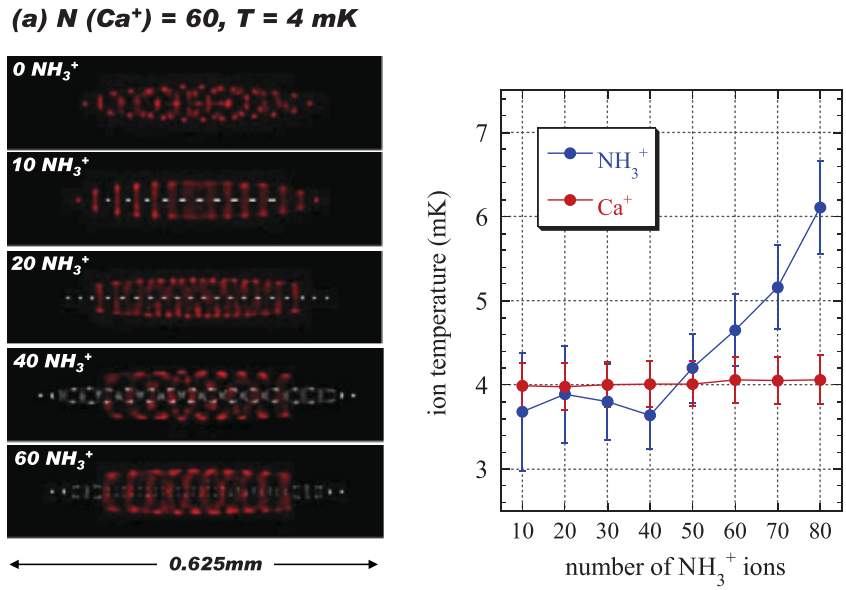

(b) $\mathrm{N}\left(\mathrm{Ca}^{+}\right)=200, \mathrm{~T}=4 \mathrm{mK}$
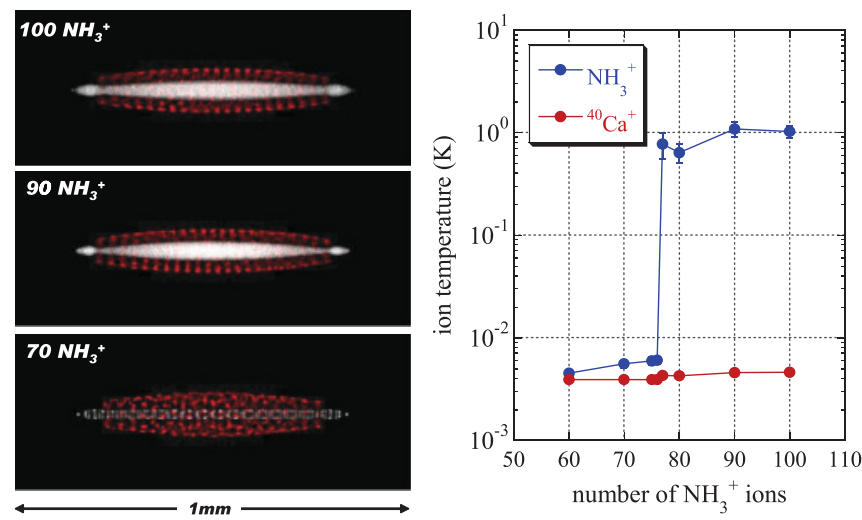

FIG. 12. (Color online) Simulation images of sympathetically cooled $\mathrm{NH}_{3}{ }^{+}$ions by laser-cooled $\mathrm{Ca}^{+}$ions for different number of $\mathrm{NH}_{3}{ }^{+}$ions: (a) $N_{\mathrm{LC}}=60$, (b) $N_{\mathrm{LC}}=200$. The trapping condition is the same as Fig. 11. The graphs show the secular temperature of each ion species as a function of $N_{\mathrm{SC}}$. The error bars show the standard deviation of the average temperatures. In (b), most error bars are smaller than the plot symbols and not indicated. The molecular ions in the center are indicated in white.

comparable to that of the $\mathrm{Ca}^{+}$ions within the uncertainties, which are $0.5 \mathrm{~K}$ for $\mathrm{NH}_{3}{ }^{+}$and $0.3 \mathrm{~K}$ for $\mathrm{Ca}^{+}$, respectively. As $N_{\text {SC }}$ increases, the $\mathrm{Ca}^{+}$crystal forms a hollow cylinder whose inside is occupied by the $\mathrm{NH}_{3}{ }^{+}$ions. As shown in the graphs of Fig. 12, the temperature of the $\mathrm{NH}_{3}{ }^{+}$ions is dependent on $N_{\mathrm{SC}}$ itself, while the temperature of the $\mathrm{Ca}^{+}$ions is nearly constant.

On the other hand, the $\mathrm{NH}_{3}{ }^{+}$ions are not sympathetically crystallized for $N_{\mathrm{SC}}>80$ and $N_{\mathrm{LC}}=200$, as shown in Fig. 12(b), even though the ratio of $N_{\mathrm{SC}}$ and $N_{\mathrm{LC}}$ is the same as Fig. 12(a). The realization of the sympathetic crystallization is therefore dependent on the total number of ions $\left(N_{\mathrm{SC}}+\right.$ $N_{\mathrm{LC}}$ ) as well as the ratio. When $N_{\mathrm{SC}}<77$, the bicrystal is successfully produced, and $T_{\mathrm{SC}}$ decreases to the comparable temperature of $T_{\mathrm{LC}}$.

When the temperature of the virtual light atoms is systematically changed from $2 \mathrm{mK}$ to $8 \mathrm{mK}$ for the same numbers of $\mathrm{Ca}^{+}$and $\mathrm{NH}_{3}{ }^{+}$ions $\left(N_{\mathrm{SC}}=N_{\mathrm{LC}}=60\right)$, we obtain the bicrystal images shown in Fig. 13(a)-13(d). As the $\mathrm{Ca}^{+}$ion temperature increases, the central part of the $\mathrm{Ca}^{+}$image becomes
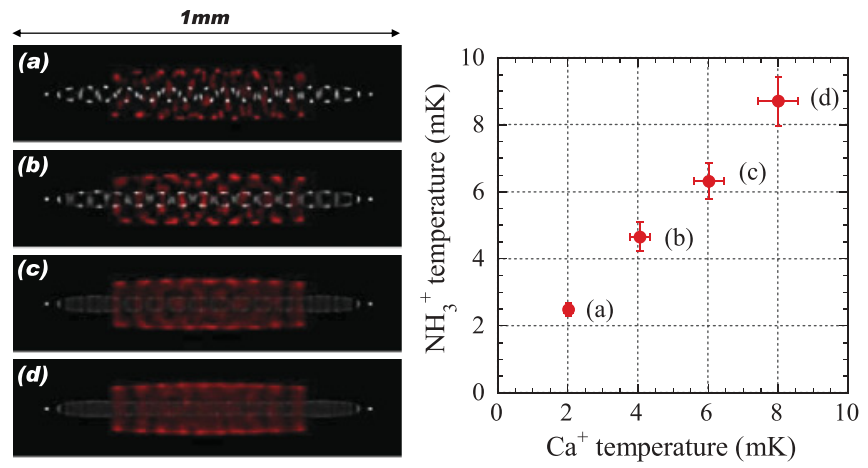

FIG. 13. (Color online) Simulation images of sympathetic crystallized $60 \mathrm{NH}_{3}{ }^{+}$ions by laser-cooled $60^{40} \mathrm{Ca}^{+}$ions produced by different temperatures of the virtual very light atoms. The graph indicates the correlation between the $\mathrm{Ca}^{+}\left(T_{\mathrm{LC}}\right)$ and $\mathrm{NH}_{3}{ }^{+}$temperature $\left(T_{\mathrm{SC}}\right)$. The points (a)-(d) in the plot correspond to the images of (a)-(d). The error bars show the standard deviation of the average temperatures. The trapping conditions are the same as in Fig. 11.

washed out. The graph of Fig. 13 is the correlation of the temperatures of both types of ion crystals. It is clear that there is a linear dependence at least in this low-temperature area, and the temperature of the sympathetically cooled molecular ions is reflected in a simulated image of laser-cooled ions. Thus the present results show that the temperature of sympathetically crystallized ions can be deduced from a CCD image of a laser-cooled ion crystal by interpreting the MD simulations.

The sympathetic crystallization of lighter ions, such as $\mathrm{H}_{2}{ }^{+}$and $\mathrm{He}^{+}$[39], never succeeded in the present simulation using $\mathrm{Ca}^{+}$coolant ions, since the difference between the trapping parameters is very large. To systematically show this phenomenon, the MD simulations of sympathetic cooling of $20 \mathrm{M}^{+}$ions by $60{ }^{40} \mathrm{Ca}^{+},{ }^{24} \mathrm{Mg}^{+}$, and ${ }^{9} \mathrm{Be}^{+}$cooled ions were performed. The upper and lower graphs of Fig. 14 show temperatures of coolant and sympathetically cooled ions, respectively, versus the mass number of sympathetically cooled ions. We find that there is a lower limit of the ion mass to achieve the sympathetic crystallization by each coolant ion. These limits possibly vary with the numbers of coolant and sympathetically cooled ions. The bumps of the upper graph (for example, the bump around the mass number 10 of the $\mathrm{Ca}^{+}$ case) correspond to the shoulders of the lower graph, where the sympathetic cooling becomes effective and the molecular ions heat the coolant ions. It is to be noted that the rf heating effect is not included in Fig. 14. Thus, it is expected that an experimental $T_{\mathrm{SC}}$ is much higher than that of Fig. 14(b) for noncrystallized light sympathetically cooled ions.

To summarize, the present simulation indicates that it is possible to determine the number of $\mathrm{NH}_{3}{ }^{+}$ions that are included inside hollow cylinder $\mathrm{Ca}^{+}$crystals by compared observed images with simulated ones especially for relatively small ion crystals. The possible accuracy is at least \pm 10 ions in Fig. 12. Note that a visible difference of the simulated images is dependent on the temperature of ions, the trapping voltages, and the numbers of ions. In an experiment, the accuracy might be limited by the spatial resolution of an observed CCD image. We also show that it is possible to deduce the ion 

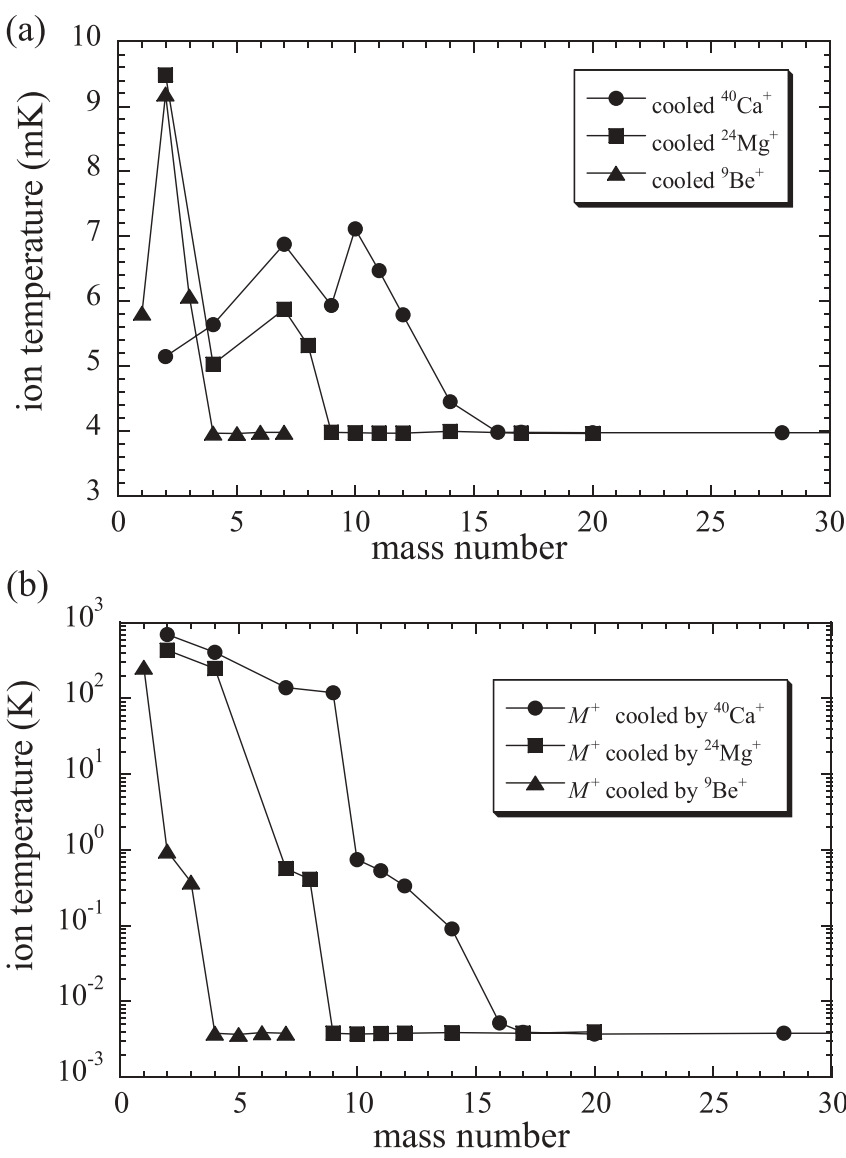

FIG. 14. Sympathetic cooling of $20 \mathrm{M}^{+}$ions by $60{ }^{40} \mathrm{Ca}^{+},{ }^{24} \mathrm{Mg}^{+}$, or ${ }^{9} \mathrm{Be}^{+}$cooled ions, respectively. The graphs show temperatures of (a) coolant ions and (b) sympathetically cooled ions versus the mass number of sympathetically cooled ions. The rf parameters are $f=5.65 \mathrm{MHz}, V_{z}=2.0 \mathrm{~V}$, and $V_{\mathrm{ac}}=250 \mathrm{~V}$ for $\mathrm{Ca}^{+}, V_{\mathrm{ac}}=150 \mathrm{~V}$ for $\mathrm{Mg}^{+}$, and $V_{\mathrm{ac}}=56.3 \mathrm{~V}$ for $\mathrm{Be}^{+}$, where the trap parameter $q$ is 0.078 for each ion species. The temperature of the virtual very light atoms is set to $4 \mathrm{mK}$.

temperature of sympathetically cooled ions from the CCD image of laser-cooled ions. When needing to cool lighter atomic and molecular ions by the sympathetic cooling method, the lighter coolant ions must be used. It is worth mentioning that MD simulations and studies of the phase transition of multispecies crystals including sympathetically cooled ions are interesting research topics left for the future.

\section{v. CONCLUSION}

We have developed a simple and faster-than-usual method to simulate observed images of ion Coulomb crystals in a linear Paul trap. As a demonstration, experimentally observed $\mathrm{Ca}^{+}$ ion Coulomb crystals were systematically compared with the simulated images. In particular, we investigated the production process of planar Coulomb crystals for a small number of $\mathrm{Ca}^{+}$ions. For relatively large ion crystals, we found that ion losses occur before producing a planar Coulomb crystal. This fact suggests that a more precise arrangement of trap electrodes, the control of stray fields, and contact potentials are necessary to produce a larger crystal disk. For large ion crystals, we have successfully reproduced the CCD images, and in doing so determined the ion temperatures and the number of ions. In addition, a simulation of the sympathetic cooling of simple molecular ions has also been performed. Our straightforward simulation method is useful in characterizing the properties of ion Coulomb crystals as well as describing the dynamics of sympathetic crystallization over a wide range of numbers of ions in a linear Paul trap. We are in the process of extending the method to MD simulations in linear multipole rf ion traps. The application of this technique to the study of ultracold molecular or atomic ion-polar molecule collisions using sympathetic crystallization and a Stark velocity selector will also be performed in the near future; such sympathetically cooled ion crystals have already been detected in the cryogenic linear octupole ion trap [31].

\section{ACKNOWLEDGMENTS}

This work is financially supported in part by a Grant-in-Aid for Young Scientists (A) from the Ministry of Education, Culture, Sports, Science and Technology (MEXT) and by the Robert A. Welch Foundation under Grant No. A1546. We thank the staff of the Techno-Center of Sophia University for fabricating the linear Paul trap. We also thank Dr. Shuichi Hasegawa for useful discussions about planar Coulomb crystals in linear Paul traps.
[1] J. I. Cirac and P. Zoller, Phys. Rev. Lett. 74, 4091 (1995).

[2] D. Porras and J. I. Cirac, Phys. Rev. Lett. 96, 250501 (2006).

[3] C. Monroe, D. M. Meekhof, B. E. King, W. M. Itano, and D. J. Wineland, Phys. Rev. Lett. 75, 4714 (1995).

[4] P. O. Schmidt, T. Rosenband, C. Langer, W. M. Itano, J. C. Bergquist, and D. J. Wineland, Science 309, 749 (2005).

[5] A. L. Wolf, S. A. van den Berg, C. Gohle, E. J. Salumbides, W. Ubachs, and K. S. E. Eikema, Phys. Rev. A 78, 032511 (2008).

[6] K. Okada et al., Phys. Rev. Lett. 101, 212502 (2008).

[7] M. Herrmann, V. Batteiger, S. Knünz, G. Saathoff, Th. Udem, and T. W. Hänsch, Phys. Rev. Lett. 102, 013006 (2009).
[8] P. F. Staanum, K. Højbjerre, R. Wester, and M. Drewsen, Phys. Rev. Lett. 100, 243003 (2008).

[9] K. Højbjerre, D. Offenberg, C. Z. Bisgaard, H. Stapelfeldt, P. F. Staanum, A. Mortensen, and M. Drewsen, Phys. Rev. A 77, 030702(R) (2008).

[10] B. Roth, J. C. J. Koelemeij, H. Daerr, and S. Schiller, Phys. Rev. A 74, 040501(R) (2006).

[11] U. Fröhlich, B. Roth, P. Antonini, C. Lämmerzahl, A. Wicht, and S. Schiller, Lect. Notes Phys. 648, 297 (2004).

[12] J. D. Weinstein, R. deCarvalho, T. Guillet, B. Friedrich, and J. M. Doyle, Nature (London) 395, 148 (1998). 
[13] R. Wynar, R. S. Freeland, D. J. Han, C. Ryu, and D. J. Heinzen, Science 287, 1016 (2002).

[14] E. A. Donley, N. R. Claussen, S. T. Thompson, and C. E. Wieman, Nature (London) 417, 529 (2002).

[15] H. L. Bethlem, G. Berden, A. J. A. van Roij, F. M. H. Crompvoets, and G. Meijer, Phys. Rev. Lett. 84, 5744 (2000).

[16] S. A. Rangwala, T. Junglen, T. Rieger, P. W. H. Pinkse, and G. Rempe, Phys. Rev. A 67, 043406 (2003).

[17] T. Rieger, T. Junglen, S. A. Rangwala, P. W. H. Pinkse, and G. Rempe, Phys. Rev. Lett. 95, 173002 (2005).

[18] M. Kirste, B. G. Sartakov, M. Schnell, and G. Meijer, Phys. Rev. A 79, 051401(R) (2009).

[19] S. Willitsch, M. T. Bell, A. D. Gingell, S. R. Procter, and T. P. Softley, Phys. Rev. Lett. 100, 043203 (2008).

[20] V. Wakelam, E. Herbst, and F. Selsis, Astron. Astrophys. 444, 883 (2005); 451, 551 (2006).

[21] J. P. Schiffer, Phys. Rev. Lett. 70, 818 (1993).

[22] H. Totsuji, T. Kishimoto, C. Totsuji, and K. Tsuruta, Phys. Rev. Lett. 88, 125002 (2002).

[23] A. Ostendorf, C. B. Zhang, M. A. Wilson, D. Offenberg, B. Roth, and S. Schiller, Phys. Rev. Lett. 97, 243005 (2006).

[24] C. B. Zhang, D. Offenberg, B. Roth, M. A. Wilson, and S. Schiller, Phys. Rev. A 76, 012719 (2007).

[25] K. Okada, K. Yasuda, T. Takayanagi, M. Wada, H. A. Schuessler, and S. Ohtani, Phys. Rev. A 75, 033409 (2007).
[26] I. M. Buluta, M. Kitaoka, S. Georgescu, and S. Hasegawa, Phys. Rev. A 77, 062320 (2008).

[27] I. M. Buluta and S. Hasegawa, J. Phys. B 42, 154004 (2009).

[28] D. H. E. Dubin, Phys. Rev. Lett. 71, 2753 (1993).

[29] W. C. Swope, H. C. Andersen, P. H. Berens, and K. R. Wilson, J. Chem. Phys. 76, 637 (1982).

[30] R. J. Clark, T. Lin, K. R. Brown, and I. L. Chuang, J. Appl. Phys. 105, 013114 (2009).

[31] K. Okada, T. Takayanagi, M. Wada, S. Ohtani, and H. A. Schuessler, Phys. Rev. A 80, 043405 (2009).

[32] L. D. Landau and E. M. Lifshitz, Mechanics (Course of Theoretical Physics) (Elsevier, New York, 1969).

[33] V. L. Ryjkov, X. Z. Zhao, and H. A. Schuessler, Phys. Rev. A 71, 033414 (2005).

[34] L. Spitzer, Physics of Fully Ionized Gases (Wiley, New York, 1962).

[35] SIMION 3D Version 8.0, Scientific Instrument Services Inc., Idaho National Laboratory.

[36] M. Block, A. Drakoudis, H. Leuthner, P. Seibert, and G. Werth, J. Phys. B 33, L375 (2000).

[37] S. L. Gilbert, J. J. Bollinger, and D. J. Wineland, Phys. Rev. Lett. 60, 2022 (1988).

[38] A. Drakoudis, M. Sollner, and G. Werth, Int. J. Mass Spectrom. 252, 61 (2006).

[39] B. Roth, U. Fröhlich, and S. Schiller, Phys. Rev. Lett. 94, 053001 (2005). 